\title{
Défaut de thermogenèse et obésité
}

\begin{abstract}
A côté de l'hyperphagie, des défauts de thermogenèse interviennent dans les mécanismes des obésités. Le tissu adipeux brun est l'effecteur principal de la thermogenèse facultative chimique, qui semble perturbée dans plusieurs types d'obésité chez les animaux de laboratoire; son rôle et son intervention réelle dans l'obésité humaine restent à préciser.
\end{abstract}

\section{Josiane Seydoux \\ Privat-docent, faculté de médecine de Genève}

J. Seydoux : département de physiologie, centre médical universitaire, 9 , avenue de Champel / 1 , rue Michel-Servet, 1211 Genève 4, Suisse. l est actuellement admis que l'obésité ne résulte pas obligatoirement d'un apport énergétique excédentaire par rapport à la moyenne des sujets de poids normal, ainsi que de nombreuses enquêtes alimentaires l'ont démontré. Mais il n'est pas encore clairement établi pourquoi, parmi des individus ayant un poids corporel, un apport alimentaire et une activité physique semblables, certains maintiennent leur poids constant et d'autres pas. L'explication de cette différence doit donc être recherchée dans les variations des diverses composantes de la dépense énergétique.

La figure 1 représente la relation entre la temperature ambiante et la dépense énergétique. Cette dernière peut être subdivisée en trois composantes: le métabolisme basal (MB), l'activité physique et la thermogenèse $(T)$. La dépense énergétique basale mesurée en situation de thermoneutralité permet le maintien des gradients ioniques, de l'activité cardiaque, respiratoire et rénale et inclut l'énergie utilisée pour le turnover des protéines. Fonction de la masse maigre, de l'âge et du sexe, elle est influencée par l'état thyroïdien et représente environ les trois quarts de la dépense totale. L'activité physique de la plupart des individus ne représente qu'une faible proportion de la dépense totale, mais peut varier considérablement en fonction de la durée et de l'intensité de l'exercice. La troisième composante, la thermogenèse, dépend chez l'homme essentiellement de la prise alimentaire. Chez les petits mammiferes, s'ajoute la thermogenèse induite par l'exposition au froid. Cette dépense, contrôlée par des centres localisés au niveau de l'hypothalamus et de la moelle épinière, est produite par le frisson dont l'effecteur est le muscle squelettique et par la thermogenèse sans frisson (ou chimique), dont l'effecteur principal est le tissu adipeux brun.

L'étude de la thermogenèse induite par la prise alimentaire a montré l'existence de deux composantes: une thermogenèse obligatoire due au coût énergétique de la digestion, de l'absorption et du stockage des nutriments ; une thermogenèse facultative qui représente une dépense supplémentaire due à l'activation, entre autres, du système nerveux sympathique. Après ingestion de nutriments, de glucose par exemple, la thermogenèse totale mesurée chez l'adulte est d'environ $10 \%$ du contenu énergétique 


\section{RÉFÉRENCES}

1. Pittet $\mathrm{Ph}$, Gygax $\mathrm{PH}$, Jéquier $\mathrm{E}$. Thermic effect of glucose and amino acids in man studied by direct or indirect calorimetry. $\mathrm{Br} J \mathrm{Nutr}$ $1974 ; 31: 343-9$

2. Flatt JP. The biochemistry of energy expenditure. In : Bray G, ed. Recent Advances in Obesity Research II. London: Newman, 1978 . 211-8.

3. Foster DO, Frydman ML. Nonshivering thermogenesis in the rat, II. Measurements of blood flow with microspheres point to brown adipose tissue as the dominant site of the calorigenesis induced by noradrenaline. Can $J$ Physiol Pharmacol 1978 ; $56: 110-22$.

4. Rothwell NJ, Stock MJ. A role for brown adipose tissue in diet-induced thermogenesis. Nature $1979 ; 218: 31-5$.

5. Girardier L. Brown fat on energy dissipating tissue. In : Girardier L, Stock J, eds. Mammalian Thermogenesis. London : Chapman et Hall, 1983 ; 50-98.

6. Ricquier D. Thermogenèse et obésité : mécanismes moléculaires. médecine/sciences 1985 ; 1 : 147-53.

7. Mory G, Bouillaud F, Combes-George M, Ricquier D. Noradrenaline controls the concentration of the uncoupling protein in brown adipose tissue. FEBS Letl 1984; 166 : 393-6.

8. Girardier L, Seydoux J. Neural control of brown adipose tissue. In : Trayhurn $\mathrm{P}$, Nicholls DG, eds. Brown Adipose Tissue. London : Arnold, 1986 : 122-51.

9. Bray GA, York DA. Hypothalamic and genetic obesity in experimental animals : an autonomic and endocrine hypothesis. Physiol Rev 1979 ; 59 : 719-809.

10. Himms-Hagen J. Obesity may be due to a malfunctioning of brown fat. Canadian Medical Association Journal 1979; 121 : 1361-4.

11. Trayhurn P. Brown adipose tissue and energy balance. In : Trayhurn P, Nicholls DG, eds. Brown Adipose Tissue. London : Arnold, 1986 ; 299-338.

12. Seydoux J, Assimacopoulos-Jeannet F, Jeanrenaud B, Girardier L. Alterations of brown adipose tissue in genetically (ob/ob) mice. I. Demonstration of loss of metabolic response to nerve stimulation and catecholamines and its partial recovery after fasting or cold adaptation. Endocrinology $1982 ; 110$ :

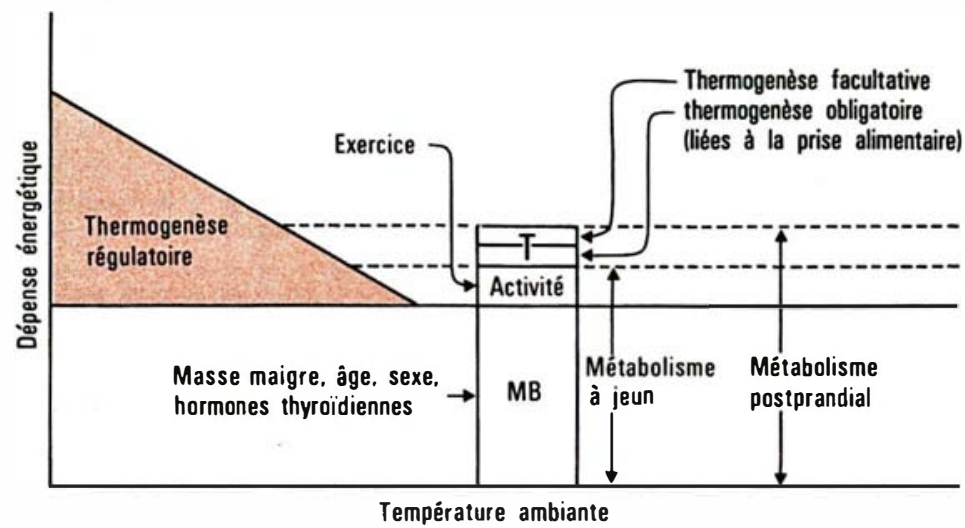

Figure 1. Diagramme illustrant la dépense énergétique et ses composantes chez l'homéotherme en fonction de la température ambiante. MB = métabolisme basal.

ingéré [1], alors que la thermogenèse obligatoire calculée à partir des données biochimiques du coût théorique de la conversion du glucose en glycogène est d'environ $5 \%$ [2]. La thermogenèse facultative liée au métabolisme du glucose représente donc la moitié de la réponse totale.

\section{Thermogenèse liée à la prise alimentaire}

Quel est l'effecteur de la thermogenèse liée à la prise alimentaire ? Le tissu adipeux brun est connu depuis 1962 pour jouer un rôle dans la thermogenèse chimique induite par le froid. Il en est également l'effecteur principal chez tous les mammifères nouveau-nés, y compris l'homme, et chez le rat adulte acclimaté au froid [3]. Depuis 1979, il est également considéré comme un effecteur de la thermogenèse liée à la prise alimentaire [4]. Ainsi, chez le petit mammifere, ces deux types de thermogenèse ont un effecteur commun. Le calcul montre que la capacité thermogène du tissu adipeux brun est de 400 watts par $\mathrm{kg}$ lorsqu'elle est stimulée au maximum soit par exposition au froid, soit par injection de noradrénaline [5]. Cette capacité de produire de la chaleur représente un court-circuit entre apport et dissipation d'énergie. Ainsi, lorsque ce tissu est activé, il devient capable d'opposer une résistance à de chaleur une partie de l'énergie ingérée. Cette capacité est contrôlée directement par l'activation du système nerveux sympathique qui, innervant dans ce tissu les adipocytes bruns et les artérioles, induit des modifications morphologiques et fonctionnelles. La séquence des événements conduisant à la production de chaleur est bien connue et décrite en détail dans l'article de D. Ricquier [6]. En bref, celle-ci débute par la liaison de la noradrénaline (libérée par les terminaisons nerveuses) à des récepteurs de type $\beta_{1}{ }^{*}$ et se termine par l'oxydation des acides gras. L'oxydation des substrats est couplée à la synthèse d'ATP dans tous les tissus, à l'exception de la graisse brune dont les mitochondries possèdent un mécanisme unique de dissipation d'énergie.

* On distingue deux types de récepteurs adréner giques $\alpha$ et $\beta$, subdivisés en $\alpha_{1}, \alpha_{2}, \beta_{1}$ et $\beta_{2}$. La liaison de la noradrénaline ou de l'adrénaline induit la modification de seconds messagers: synthèse de l'AMP cyclique par l'adénylate cyclase pour les récepteurs $\beta_{1}$ et $\beta_{2}$, augmentation de la concentration cytosolique de calcium ionisé pour les récepteurs $\alpha_{1}$, diminution de la synthèse d'AMP cyclique par inhibition de l'adénylate cyclase pour les récepteurs $\alpha_{2}$. l'obésité, en dissipant sous forme 
Ce mécanisme localisé dans la membrane mitochondriale consiste en un court-circuit à protons qui, dissociant l'oxydation des substrats de la synțhèse stoechiométrique d'ATP, permet à la respiration de procéder à grande vitesse pour ne produire que de la chaleur. Les acides gras jouent un rôle essentiel dans la thermogenèse, non seulement comme substrats mais encore comme activateurs des canaux à protons par l'intermédiaire de la protéine découplante. Celle-ci, de poids moléculaire 32000 , n'existe que dans le tissu adipeux brun; sa synthèse est augmentée lorsque ce tissu est stimulé [7].

Dans la séquence des événements conduisant à la production de chaleur, il faut souligner l'importance de la répartition du débit cardiaque. En effet, la stimulation du taux métabolique des cellules brunes nécessite un apport accru de substrat et d'oxygène, ainsi qu'une augmentation de la capacité de transfert de la chaleur produite. Ces trois phénomènes doivent, en quelque sorte, être coordonnés pour éviter soit un déficit de substrat ou d'oxygène, soit une surchauffe du tissu. Cette coordination entre la régulation du débit sanguin et l'activation du métabolisme cellulaire est essentiellement d'origine nerveuse [8]. L'administration de noradrénaline [3], comme toute situation dans laquelle le système nerveux sympathique est activé, induit une augmentation du débit sanguin dans le tissu adipeux brun par un mécanisme encore non élucidé. En effet, aucun récepteur ßadrénergique vasodilatateur n'a été mis en évidence dans les vaisseaux de ce tissu. Les effets de la noradrénaline sont vraisemblablement indirects et secondaires à l'activation métabolique ou à la libération d'une substance restant encore à identifier.

\section{Thermogenèse et obésité}

Les modèles animaux d'obésité, que celle-ci soit d'origine génétique ou induite par lésion des noyaux hypothalamiques ventro$m / s n^{\circ} 7$ vol. 3, seplembre 87

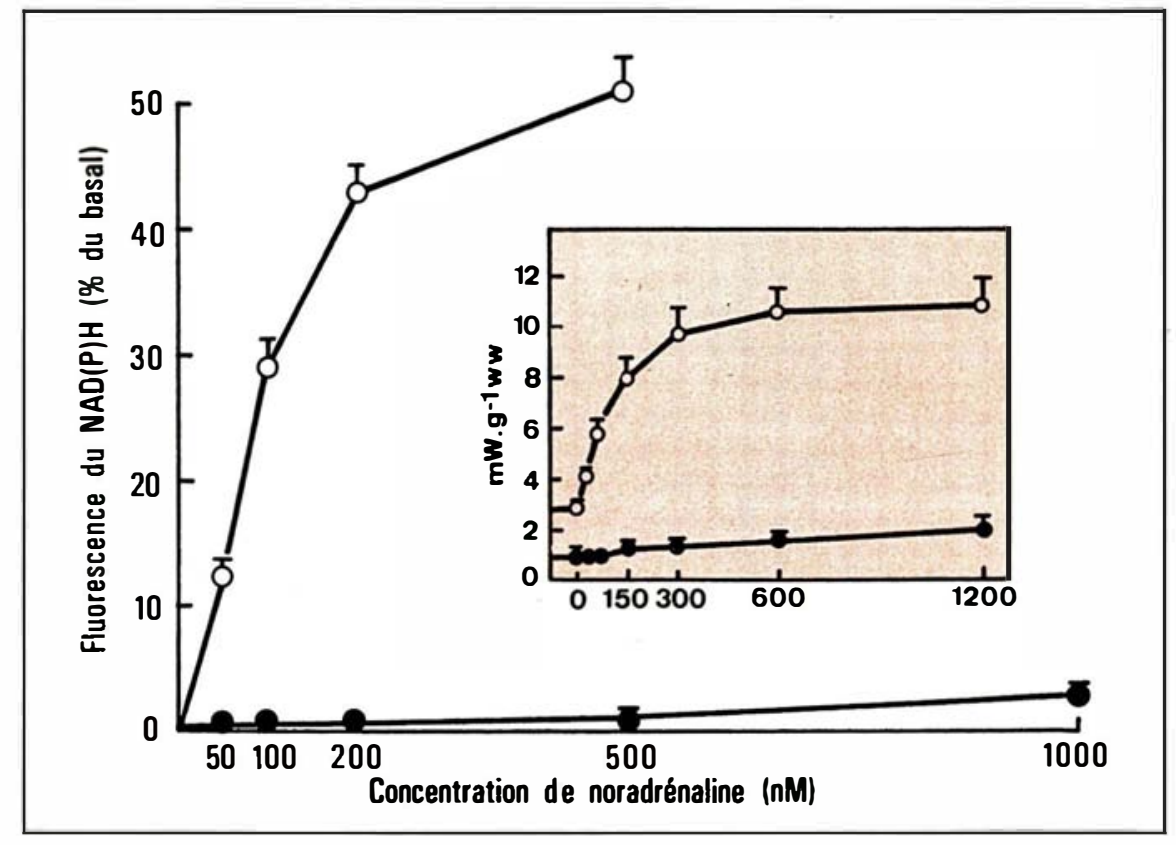

Figure 2. Effets de concentrations croissantes de noradrénaline sur le taux métabolique de tissu adipeux brun interscapulaire de souris ob/ob (o) et de témoins (0). Les index mesurés sont (a) le niveau rédox des pyridines nucléotides lune augmentation de la fluorescence émise correspond à un apport accru d'équivalents réduits à la chaine respiratoire) et (b) la production de chaleur, basale et stimulée, mesurée en calorimétrie directe (encadré). II faut noter la très faible réponse thermogénique du tissu de l'obèse (figure extraite de [12]).

médians (VMH), présentent tous, mais à des degrés divers, un défaut de thermogenèse dans leur réponse à l'exposition au froid ou à la prise alimentaire. Plusieurs articles de revue récents [9-11], comparant les différents modèles, rassemblent une abondante bibliographie sur ce sujet.

Bien que l'hyperphagie soit une des caractéristiques de la souris $o b / o b$ et du rat VHM, elle n'est cependant pas indispensable au développement de l'obésité, ce qui suggère qu'une composante au moins de la dépense énergétique est diminuée. Lorsque les souris $o b / o b$ sont exposées à $4^{\circ} \mathrm{C}$, elles meurent d'hypothermie en quelques heures par défaut d'activation de la thermogenèse sans frisson. Des travaux effectués dans notre laboratoire ont contribué à démontrer que, chez la souris $o b / o b$ et le rat VMH, la capacité métabolique du tissu adipeux brun était fortement diminuée : aussi bien l'activation directe du système nerveux sympathique par stimulation de ses nerfs que la stimulation indirecte induite par la noradrénaline, sont réduites [12]. Les effets de concentrations croissantes de noradrénaline sur la réponse métabolique de tissu adipeux brun interscapulaire de souris $o b / o b$ et de leurs témoins adaptés à $23^{\circ} \mathrm{C}$ sont représentés sur la figure 2. La capacité du tissu de la souris $o b / o b$ à produire de la chaleur à l'état basal est diminuée, et encore plus fortement lors de stimulation par la noradrénaline. Cependant, ce défaut d'activation métabolique n'est pas irréversible : dans certaines conditions, cette capacité peut être partiellement rétablie après acclimatation graduelle au froid ou mise au jeûne de la souris. La séquence d'événements conduisant à la production de chaleur a été étudiée afin de localiser la ou les étapes responsables de cette absence de réponse. Il a été ainsi montré que plusieurs d'entre elles sont impliquées : le nombre de récepteurs $ß$ est augmenté, l'acti- 


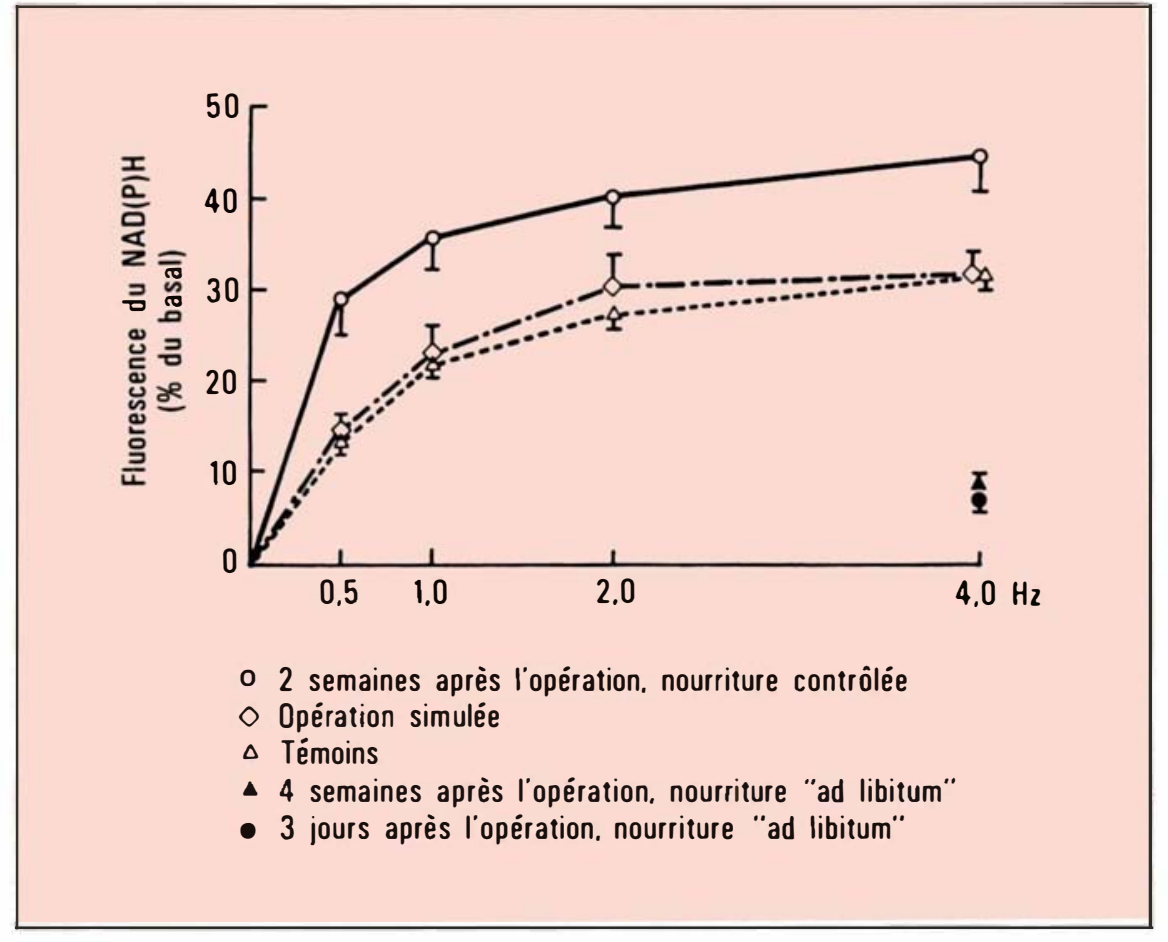

\section{RÉFÉRENCES}

13. Ashwell M, Wells C, Dunnett SB. Brown adipose tissue: contributions of nature and nurture to the obesity of an obese mutant mouse (ob/ob) Int J Obes 1986 ; 10 : 355-73.

14. Seydoux J, Ricquier D, RohnerJeanrenaud F, at al. Decreased guanine nucleotide binding and reduced equivalent production by brown adipose tissue in hypothalamic obesity. Recovery after cold acclimation. FEBS Lett $1982 ; 146$ : 161-4.

15. Silva JE, Larsen PR. Potential of brown adipose tissue type II thyroxine 5'-deiodinase as a local and systemic source of triiodothyronine in rats. $J$ Clin Invest 1985; 76 : 2296-305.

16. Bianco AC, Silva JE. Intracellular conversion of thyroxine to triiodothyronine is required for the optimal thermogenic function of brown adipose tissue. $J$ Clin Invest 1987 ; 79 : 295-300.

17. Kates AL, Himms-Hagen J. Defective cold-induced stimulation of thyroxine 5'deiodinase in brown adipose tissue of the genetically obese (ob/ob) mouse. Biochem Biophys Res Commun 1985 ; 130 : 188-93.

18. Shibata M, Benzi R, Seydoux J, Girardier L. Hyperthermia induced by pre-pontine knife-cut : evidence for a tonic inhibition of nonshivering thermogenesis in anesthetized rat. Brain Res 1987 (sous presse).

19. Rotwell NJ, Stock MJ, Thexton AJ. Decerebration activates thermogenesis in the rat. $J$ Physiol (Lond) 1983; 342 : 15-22.

20. Joy RTJ. Responses of cold-acclimatized men to inf used norepinephrine. J Appl Physiol $1963 ; 18$ : 1209-12.

21. Jessen K. An assessment of human regulatory noushivering thermogenesis. Acta Anaesthesiol Scand 1980; $24: 138-43$.

22. Astrup A. Thermogenesis in human brown adipose tissue and skeletal muscle induced by sympathomimetic stimulation. Acta Endocrinol

Figure 3. Stimulation électrique de I'innervation du tissu adipeux brun interscapulaire chez le rat rendu obèse par lésion des noyaux hypothalamiques ventromédians : effets sur l'état rédox des pyridines nucléotides. II faut noter (a) la faible réponse obtenue à $4 \mathrm{~Hz}$ sur des tissus prélevés trois jours ou quatre semaines après la lésion, (b) la réponse légèrement supérieure à celle des témoins observée lorsque les animaux lésés ont reçu pendant deux semaines la même quantité de nourriture que les témoins.

vité, sous stimulation, de l'adénylate cyclase n'est pas changée, alors que la lipolyse et l'oxydation des acides gras sont diminuées, de même que le taux de protéine découplante, le turnover de noradrénaline (index de l'activation du système nerveux sympathique), ainsi que le débit sanguin du tissu adipeux brun. L'acclimatement de la souris obèse au froid permet une normalisation plus ou moins complète de tous ces paramètres. Une étude récente visant à découvrir l'existence possible d'un défaut mitochondrial intrinsèque, au moyen de greffes de graisse brune de souris obèse sous la capsule rénale de souris témoin, a montré que la diminution de la capacité de thermogenèse n'est pas le résultat d'un défaut intrinsèque ; en effet la transplantation dans un "environnement normal " est capable de transformer les adipocytes bruns de souris "obèses " en cellules "normales" [13].

Quant à la capacité thermogène des adipocytes bruns de rats VMH, elle aussi est diminuée lorsqu'on stimule son innervation, comme l'illustre la figure 3 : la réponse obtenue, à la fréquence maximale de $4 \mathrm{~Hz}$ n'est que le quart de celle des témoins 3 jours ou 4 semaines après la lésion; cependant, elle est normalisée si le rat obèse reçoit la même quantité de nourriture que les témoins ou s'il est adapté au froid [14]. Bien que leur tissu adipeux brun présente qualitativement les mêmes anomalies que celles décrites chez la souris obèse, les rats $\mathrm{VMH}$, eux, survivent à l'exposition au froid, ce qui indique que leur thermogenèse chimique n'est pas altérée. Cependant, la thermogenèse liée à la prise de nourriture n'est pas activée chez ces rats hyperphagiques. Ainsi, la 
diminution de la capacité de production de chaleur par la graisse brune peut expliquer, en partie au moins, l'obésité de ces rongeurs.

\section{Hormones thyroïdiennes et thermogenèse régulatoire}

Il était admis jusque très récemment que les hormones thyroïdiennes $\left(\mathrm{T}_{3}\right.$ et $\left.\mathrm{T}_{4}\right)$ n'exerçaient qu'un effet permissif vis-à-vis de l'action de la noradrénaline sur le tissu adipeux brun. On vient de montrer que ce tissu, lorsqu'il est stimulé, peut constituer une source plasmatique de $T_{3}[15]$. En effet, il est l'un des effecteurs de la transformation périphérique de $T_{4}$ en $T_{3}$, source principale de $\mathrm{T}_{3}$ circulante, grâce à son contenu en enzyme de conversion, la thyroxine 5'-désiodase dont l'activité est fortement augmentée par le système sympathique. De plus, la production de $\mathrm{T}_{3}$ dans le tissu adipeux brun est indispensable à une expression optimale de la protéine découplante [16]. Ainsi, la 5'-désiodase des adipocytes bruns est un élément clé du fonctionnement normal de ceux-ci, ainsi que l'atteste le rôle direct de la $T_{3}$ dans la synthèse de la pro- téine découplante. Or, cette 5'désiodase du tissu adipeux brun de souris obèses n'est que très faiblement activée (deux fois seulement au lieu de 26 fois pour les témoins) lors de leur exposition au froid [17]. Ainsi, l'absence de stimulation de cette enzyme peut contribuer de façon significative chez ces animaux obèses à la réduction de la capacité du tissu adipeux brun à produire de la chaleur.

\section{Un centre inhibiteur de la production de chaleur}

Chez le rat anesthésié, une section stéréotaxique (fine, très localisée, faite au microcouteau) dans la région prépontique (figure 4), induit une élévation stable de 3 à $4{ }^{\circ} \mathrm{C}$ de la température du côlon, sans frisson ni augmentation visible du tonus musculaire [18]. Les mesures de la répartition du débit cardiaque et de la température du tissu adipeux brun interscapulaire indiquent une augmentation massive de la production de chaleur dans ce tissu (figure 5). D'une analyse plus poussée, on peut inférer que c'est le tissu adipeux brun qui est la source de l'hyper-

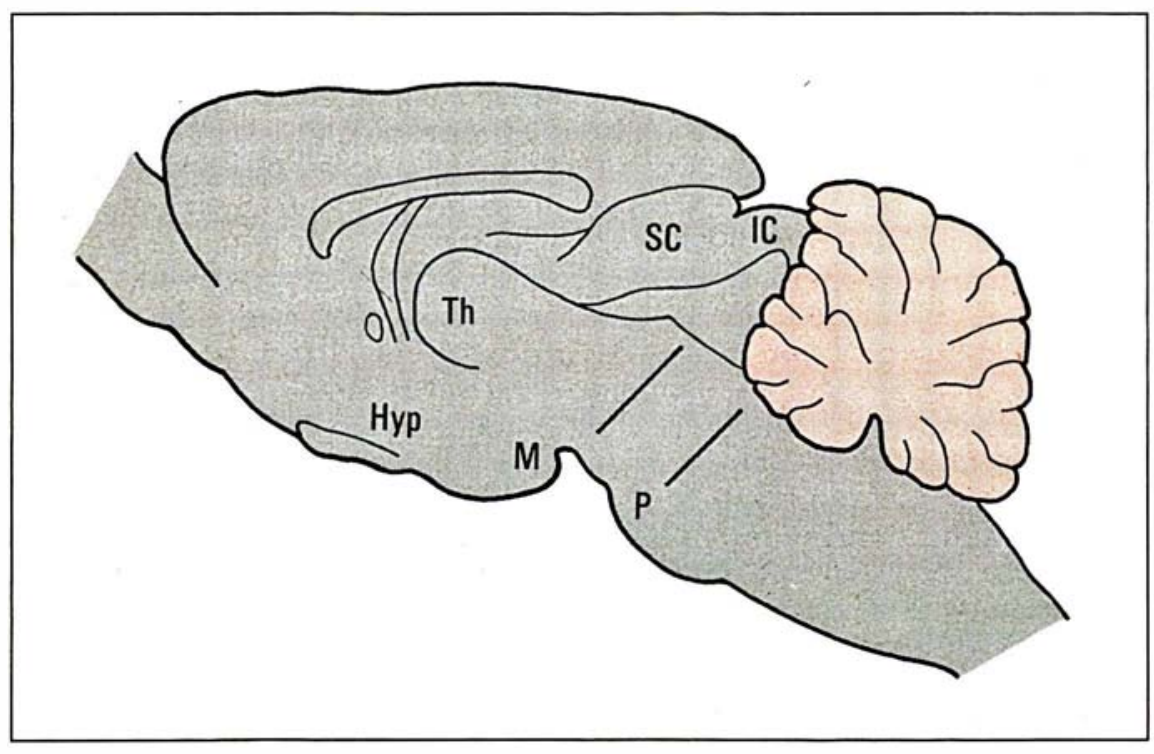

Figure 4. Coupe sagittale de cerveau de rat illustrant l'emplacement de lésions faites, à l'aide d'un microcouteau monté sur un appareil stéréotaxique, aux niveaux prépontique ( $P=$ pont) et post-mamillaire $(M=$ corps mamillaire). $S C=$ colliculus supérieur; $T h=$ thalamus; Hyp = hypothalamus; $I C=$ colliculus inférieur.

$\mathrm{m} / \mathrm{s} n^{\circ} 7 \mathrm{vol}$. 3, septembre 87 thermie. En effet, la température s'élève beaucoup plus rapidement dans ce tissu que dans le côlon et elle se stabilise à un niveau plus élevé, sans que soit changée notablement la vasomotricité. Le traitement par un ß-bloquant confirme cette conclusion. Une section localisée un peu plus haut dans la région mésencéphalique, en revanche, reste sans effet. Chez le rat génétiquement obèse $(f a / f a)$, la décérébration ne change pas significativement la température du tissu adipeux brun, donc du côlon, contrairement au témoin qui devient hyperthermique [19]. Ces résultats suggèrent que les rats obèses ne possèdent pas ce centre responsable de l'inhibition tonique de la production de chaleur ou qu'ils présentent un défaut d'activation centrale et/ou périphérique de leur tissu adipeux brun.

\section{L'effecteur de la thermo- genèse chez l'homme}

Des sujets légèrement vêtus, acclimatés cinq semaines au froid, ont une réponse métabolique accrue à une perfusion de noradrénaline [20]. De même, l'homme non adapté et exposé de façon aiguë au froid, peut sans frisson et sans inconfort visibles, par une libération accrue de noradrénaline circulante et par l'utilisation de ses lipides comme substrat principal, augmenter sa production de chaleur [21]. La noradrénaline ne serait-elle pas, comme chez l'animal, le médiateur de la thermogenèse régulatoire ?

Bien que le tissu adipeux brun soit morphologiquement présent chez l'humain, sa capacité fonctionnelle n'est pas aisée à déterminer. Les différentes localisations en fonction de l'âge sont décrites en détail dans une revue sur le sujet [22]. Cependant, des mesures biochimiques effectuées sur du tissu adipeux brun prélevé chez des patients atteints d'un phéochromocytome ou chez des travailleurs exposés aux rigueurs de l'hiver finlandais (deux situations dans lesquelles le système sympathique est stimulé) ont montré que 

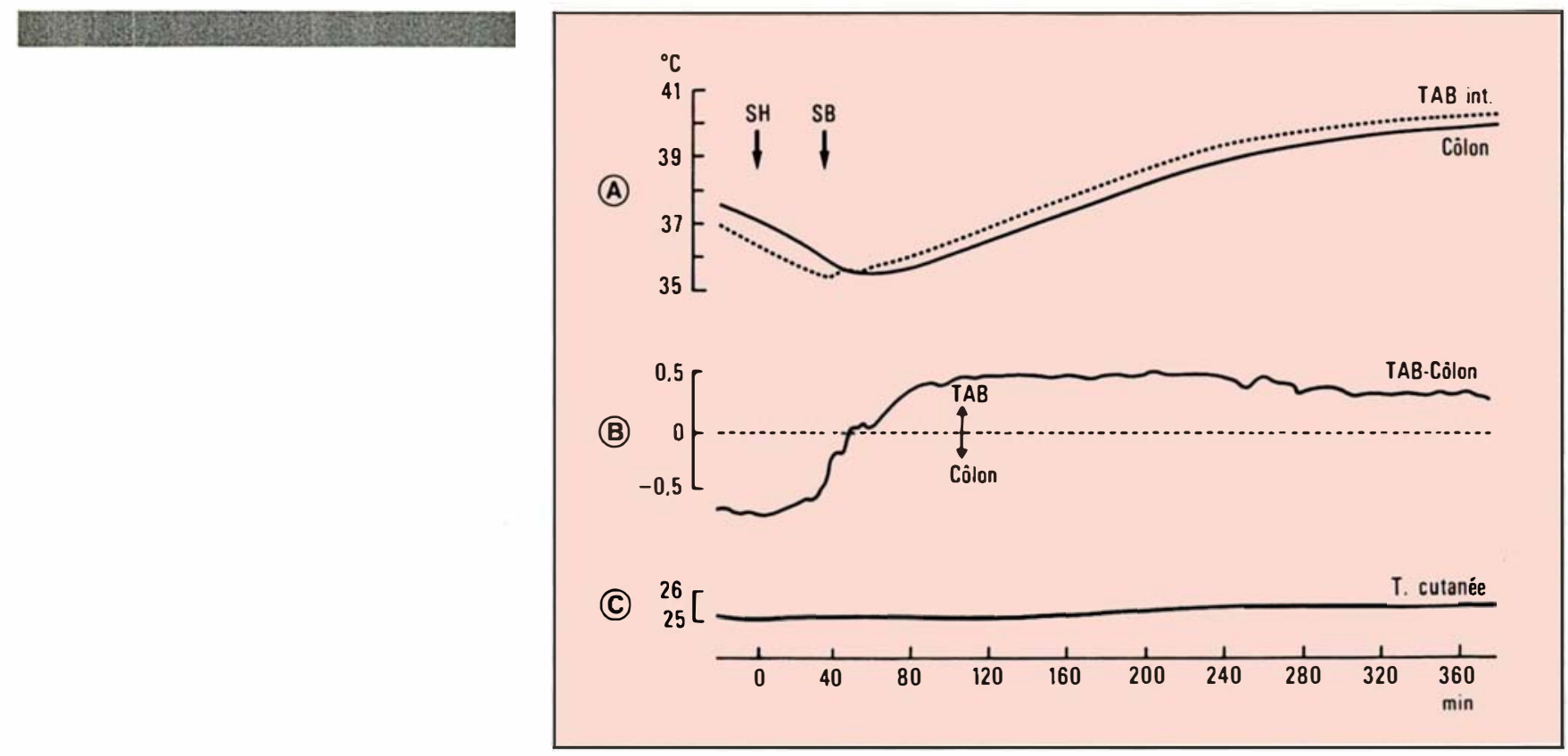

\section{RÉFÉRENCES}

23. Lever JD, Jung RT, Nnodim JO, Leslie PJ, Symons D. Demonstration of a catecholaminergic innervation in human perirenal brown adipose tissue at various ages in the adult. Anat Rec $1986 ; 215: 251-5$.

24. Lean MEJ, James WPT, Jennings G, Trayhurn $P$. Brown adipose tissue uncoupling protein content in human infants, children and adults. Clin Sci $1986 ; 71$ : 291-7.

25. Arch JRS, Ainsworth AT, Cawthorne MA, et al. Atypical B-adrenoreceptor on brown adipocytes as target for anti-obesity drugs. Nature 1984 ; 309 : 163-5.

26. Jéquier E. Energy expenditure in obesity. Clin Endocrinol Metab $1984 ; 13$ : 563-80.

27. Thorin D, Golay A, Simonson DC, Jéquier E, Felbert JP, De Fronzo RA. The effect of selective beta adrenergic blockade on glucose-induced thermogenesis in man. Metabolism 1986 ; 35 : 524-8.

28. Welle S, Lilavivat U, Campbell RG. Thermic effect of feeding in man : increased plasma norepinephrine levels following glucose but not protein or fat consumption. Metabolism 1981 ; 30 : 953-8.

29. Schutz Y, Bessard T, Jéquier E. Dietinduced thermogenesis measured over a whole day in obese and non obese women. $A m \mathrm{~J}$ Clin Nutr $1984 ; 40$ : 542-52.

30. Jung RT, Shetty PS, James WPT, Barrand MA, Callingham BA. Reduced thermo-

Figure 5. Effets, chez lo rat anesthésié, d'une section prépontique (SB) : (a) sur la température du cólon, du tissu adipeux brun interscapulaire ITAB int.) (partie A) et sur celle de la peau (patte postérieure) prise comme index de la vasomotricité (partie $C$ ), (b) sur la différence de température entre le $T A B$ interscapulaire et le cólon (partie B). La température du TAB interscapulaire augmente plus rapidement et avec un temps de latence plus court que celle du cólon, pour atteindre une valeur stable plus élevée. II faut noter que la section localisée au niveau post-mamillaire (SH) est sans effet sur l'évolution des différentes températures. La température ambiante est de $23^{\circ} \mathrm{C}$.

ce tissu possédait un équipement enzymatique semblable à celui des rongeurs. De plus, ce tissu paraît bien avoir conservé chez l'adulte une capacité fonctionnelle : on peut le supposer par la présence d'une innervation catécholaminergique du tissu adipeux brun périrénal [23] et par celle de la protéine découplante, non seulement chez le nouveau-né et l'enfant, mais aussi chez l'adulte [24]. La présence de cette protéine est un élément essentiel pour apprécier la capacité fonctionnelle des adipocytes bruns, mais sa contribution à la thermogenèse régulatoire chez l'homme demeure néanmoins difficile à évaluer, étant donnée la grande dispersion de ce tissu. Si l'on admet qu'il possède chez l'homme la même capacité que chez les rongeurs, soit 400 watts par kg, $200 \mathrm{~g}$ (parmi les $15 \mathrm{~kg}$ de lipides stockés) suffiraient au doublement du métabolisme basal, situation peu fréquente chez l'homme de nos sociétés occidentales. Il reste que le muscle squelettique de par sa masse relative importante $(50 \%)$ pourrait aussi être un effecteur : chez des sujets adultes, la moitié de l'augmentation de la thermogenèse induite par un agent sympathicomimétique, l'éphédrine, a pu en effet être attribuée à la musculature squelettique [22].

La fonction dissipative du tissu adipeux brun peut être influencée chez l'adulte par la noradrénaline et, potentiellement, par des substances visant au traitement pharmacologique de l'obésité. En effet, l'utilisation chez les rongeurs d'une série d'agonistes adrénergiques récemment dẹveloppée, a mis en évidence, dans les adipocytes blancs et bruns, la présence de récepteurs $B_{1}$ atypiques : seule la lipolyse est stimulée, alors que la fonction des autres organes cibles 
$\beta_{1}$ n'est pas affectée. De plus, l'administration quotidienne de l'un de ces agonistes à des souris $o b / o b$ a provoqué une diminution de leur prise de poids [25], ce qui ouvre des perspectives nouvelles dans le traitement de l'obésité.

Enfin, une activation généralisée du système sympathique peut être invoquée comme un des stimulants de la thermogenèse facultative postprandiale.

Lorsqu'un sujet ingère un repas, sa production de chaleur s'accroît pendant plusieurs heures. Cet effet thermique varie non seulement avec la nature des nutriments, mais aussi selon l'état nutritionnel des sujets. Il est plus élevé pour les protéines que pour les glucides et les lipides. Comme nous l'avons vu précédemment, une partie de cet effet est la thermogenèse obligatoire. Quant à la thermogenèse facultative, bien que son origine ne soit pas encore définitivement établie, sa contribution n'est pas sans importance dans la régulation du poids corporel. En effet, plusieurs études ont révélé qu'elle peut présenter un déficit chez les sujets obèses. Bien qu'elle ne représente qu'une faible part de la dépense totale, par addition d'un repas à l'autre, ces petits déficits peuvent à la longue représenter une accumulation d'énergie, c'est-à-dire un poids excédentaire. Cette question est traitée de façon très complète par E. Jéquier [26] qui, avec son équipe, a beaucoup contribué à l'étude de l'équilibre énergétique chez l'homme normal ou obèse. Plusieurs travaux mentionnent, chez des sujets de poids normal, une augmentation du taux plasmatique de noradrénaline consécutive à l'ingestion de glucides. On ne sait pas actuellement si cet accroissement de la noradrénaline est dû à l'activité spécifique de certains organes ou s'il représente une réponse sympathique généralisée, ni s'il résulte d'un effet direct ou indirect (par libération d'insuline) de la prise de glucose sur les centres impliqués dans la régulation du tonus sympathique. En utilisant un B-bloquant sélectif (le métoprolol), on a pu mon- trer, chez l'homme, que la composante liée à l'activation du système sympathique de l'effet thermique induit par le glucose, était de nature " $B_{1}$-adrénergique " [27]: Cependant, des protéines ou des lipides ingérés en quantité isocalorique au glucose ne modifient pas la concentration plasmatique de noradrénaline, alors que le taux métabolique* des sujets s'est accru [28]. Il en résulte donc que l'activation du système sympathique ne peut expliquer qu'une partie de la thermogenèse facultative.

Bien que le métabolisme à jeun (exprimé en valeur absolue) des sujets obèses soit plus élevé que celui des sujets témoins, l'augmentation de la production de chaleur induite par l'ingestion de glucides seuls, ou de repas de composition mixte est, selon les auteurs, inférieure ou semblable à celle des témoins [26]. La contradiction apparente entre ces résultats pourrait s'expliquer par la variabilité dans le choix des sujets, leur âge, la composition des repas tests, ou la durée des mesures. Une étude récente [29] a montré, chez les obèses, une diminution de l'effet thermique lié à l'ingestion de trois repas pris au cours des 24 heures de la mesure $(8,7 \%$ de l'énergie ingérée et $14,8 \%$ chez les sujets témoins) ainsi qu'une diminution de l'activité du système sympathique. De même, l'injection intraveineuse de noradrénaline n'induit chez l'obèse qu'une augmentation de $9,6 \%$ de son taux métabolique (au lieu de 21,2\% chez le témoin) ; cet accroissement plus faible n'est pas dû à un défaut de lipolyse, celle-ci se trouvant au contraire accrue [30].

Bien que l'obésité soit parfois due à une hyperphagie évidente, il n'en demeure pas moins que, dans de nombreux cas, on peut attribuer un rôle important à un déficit de thermogenèse facultative; les recherches dans ce domaine sont en pleine évolution - Taux métabolique : quantité d'énergie dissipée par
unité de temps.

\section{Summary}

This short review first describes the various components of the energy expenditure in homeotherms. It emphasizes the role of brown adipose tissue as an effector of nonshivering and diet-induced thermogenesis in small mammals and its involvement in both thermic and weight regulation. The role of the sympathetic nervous system in these thermogenic responses is also discussed. The defective brown adipose tissue activation is found in genetically or experimentally obese rodents and this may explain in part their increased food efficiency. Furthermore, optimal thermogenic function of brown adipose tissue requires intracellular conversion of thyroxine to triiodothyronine, a mechanism found to be defective in the tissue of obese rodents. Evidence of the existence of a tonic inhibitory center, located in prepontine area and acting on brown adipose tissue metabolism is presented. Finally, the possible role of brown adipose tissue as well as that of increased sympathetic nervous system activity in dietinduced thermogenesis in man is also discussed.

\section{TIRÉS A PART}

J. Seydoux : département de physiologie, centre médical universitaire, 9 , avenue de Champel / 1, rue Michel-Servet, 1211 Genève 4, Suisse. 